\title{
Application of Homotopy Perturbation Method to a Viscous Incompressible Flow Problem through an Orifice
}

\author{
Abedlkader GASMI*, Afaf SALHI \\ Laboratory of Pure and Applied Mathematics, Faculty of mathematics and computer science, M'sila University,Algeria \\ *Corresponding Author: gasmi_a@yahoo.fr
}

Copyright (C)2014 Horizon Research Publishing All rights reserved.

\begin{abstract}
This paper concern with the two dimensional, steady flow problem of viscous incompressible fluid through an orifice. A problem of such type has been solved by applying Laplacian-Driver Method or LAD method given by Roache [1]. The resulting system of linear equations is solved by He's homotopy perturbation method (HPM). The obtained results give a good agreement with the previous numerical solutions which reveals the effectiveness and convenience of the homotopy perturbation method (HPM).
\end{abstract}

Keywords Stream function,Incompressible flow, Cylindrical coordinates, Navier-Stokes equations, He's HPM Method

\section{Introduction}

One of the central problems in fluid mechanic's is the study of the steady flow of a viscous incompressible fluid. Recentely; Parment and Saibel [2] solved the axisymmetric creeping flow problem through an orifice in a plane wall. Hunt [3] discussed the classical axially symmetric jet problem. He assumed an orifice in the wall of the reservoir, and adopted the approach of integral equations and applied trial and error method. In [4] and [?] we have considered the symmetrical and asymmetric flow cases of incompressible and inviscid fluid.

Several researchers have used numerical methods to solve fluid flow problems. Greenspan [6] has considered the viscous incompressible fluid through an orifice in a channel. He applied the simple smoothing process which yields diagonally dominant system of linear equations. Mittal and Sharma [7] applied the Laplacian-Driver method to solve the problem considered by Greenspan[6]. The resulting system of equations obtained after applying central difference formulate were solved by modified Hockney's method. Bulut [8] give the analytical solution of this problem using the Adomian decomposition method.

In the present work, we apply the homotopy perturbation method $[9,13]$ to solve the problems modeling the flow through an orifice in a channel considered by Greenspan [6]. The homotopy perturbation method is seen to provide a direct scheme for solving these nonlinear problems without any need for linearization or any restrictive assumptions. Moreover, the method is found to greatly reduce the size of computational work. Our interest is to compare the analytical solutions obtained by homotopy perturbation method with numerical solutions. We present a comparative study and find that HPM solutions compare well with the numerical solutions therefore prove the HPM is powerful and effective which can easily handle wide class of non-linear problems.

\section{Mathematical formulation}

Let us consider $S_{1}, S_{2}$ and $S_{3}$ three coaxial cylinders joined to form a channel with an orifice, as shown in figure1. At far downstream and at far upstream of the flow, we will assume Poiseuille conditions are valid.

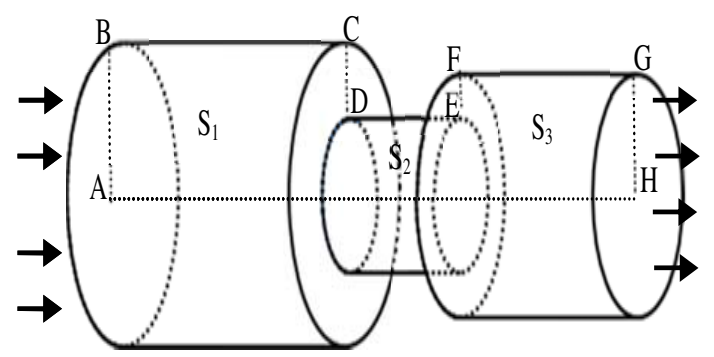

Figure 1. Sketch of the flow in a three-dimensional channel.

Inside of this channel, it will be assumed that the three dimensional Navier- Stokes equations govern the motion of the fluidGreenspan [6]. However, because of the axial symmetry, the problem can be formulated analytically as a plane and the flow domain become polygon $A B C D E F G H$, as shown in figure 2, and we use axisymmetric modeling for reducing a $3 \mathrm{D}$ model to a $2 \mathrm{D}$ plane.

On the interior of the flow domain, the equations of motion to be satisfied are the Navier Stokes equations, that is, 


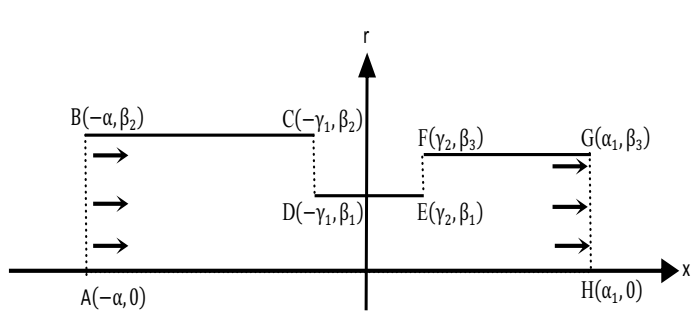

Figure 2. Sketch of the flow through an orifice.

$$
\begin{gathered}
\triangle \psi=-\zeta \\
\triangle \zeta-R\left(\frac{\partial \psi}{\partial x} \frac{\partial \zeta}{\partial y}-\frac{\partial \psi}{\partial y} \frac{\partial \zeta}{\partial x}\right)=0
\end{gathered}
$$

Here $\psi$ is the stream function, $\zeta$ is the vorticity and $R$ is the Reynolds number.

On the boundary of the domain we consider $B C D E F G$ and the $\mathrm{x}$-axis as two stream lines. Without loss of generality, we choose $\psi=1$ on the streamline $B C D E F G$. It follows from the choice of the dimensionless variables that $\psi=0$ on stream line $A H$ The boundary conditions for the domain are given as

$$
\begin{gathered}
\frac{\partial \psi}{\partial y}=0 \text { on } A H \\
\frac{\partial \psi}{\partial y}=0 \text { on } B C, D E \text { and } F G \\
\frac{\partial \psi}{\partial x}=0 \text { on } C D \text { and } E F \\
\psi=3 y^{2}-2 y^{3}, \quad \zeta=12 y-6 \text { on } A B \\
\frac{\partial \psi}{\partial x}=0, \quad \frac{\partial \psi}{\partial x}+R \frac{\partial \zeta}{\partial y}\left(\zeta+\frac{\partial^{2} \psi}{\partial y^{2}}\right), \text { on } G H
\end{gathered}
$$

Due to the axisymmetry the flow is conveniently studied in a cylindrical coordinate system with the x-axis coinciding with the axis of symmetry of the flow. Converting to cylindrical coordinates we obtain:

$$
\begin{gathered}
\frac{\partial^{2} \psi}{\partial r^{2}}-\frac{1}{r} \frac{\partial \psi}{\partial r}+\frac{\partial^{2} \psi}{\partial x^{2}}=-r^{2} \Omega \\
r\left(\frac{\partial^{2} \Omega}{\partial r^{2}}-\frac{3}{r} \frac{\partial \Omega}{\partial r}+\frac{\partial^{2} \Omega}{\partial x^{2}}\right)=R\left(\frac{\partial \psi}{\partial r} \frac{\partial \Omega}{\partial x}-\frac{\partial \psi}{\partial x} \frac{\partial \Omega}{\partial r}\right)
\end{gathered}
$$

$\Omega$ is related to the vorticity $\zeta$ by $\Omega=\zeta / r$, and the boundary conditions is given in cylindrical coordinates by:

$$
\begin{aligned}
& \psi=0, \quad \frac{\partial \psi}{\partial r}=0 \quad \text { on } \quad A H \\
& \psi=1, \quad \frac{\partial \psi}{\partial r}=0 \text { on } B C, D E \text { and } F G \\
& \psi=1, \quad \frac{\partial \psi}{\partial x}=0 \text { on } C D \text { and } E F \\
& \left\{\begin{array}{lll}
\psi=\left(\frac{r}{\beta_{2}^{2}}\right)^{2}\left[2-\left(\frac{r}{\beta_{2}^{2}}\right)^{2}\right] & \text { on } & A B \\
\Omega=\frac{8}{\left(\beta_{2}\right)^{4}} & \text { on } & A B
\end{array}\right. \\
& \left\{\begin{array}{lll}
\psi=\left(\frac{r}{\beta_{3}^{2}}\right)^{2}\left[2-\left(\frac{r}{\beta_{3}^{2}}\right)^{2}\right] & \text { on } & G H \\
\Omega=\frac{8}{\left(\beta_{3}\right)^{4}} & \text { on } & G H
\end{array}\right.
\end{aligned}
$$

\section{Analysis of HE's homotopy perturbation method}

The steady two-dimensional motion of a viscous fluid in a tube is considered. This problem is equations of motion which govern the flow field in the tube are the Navier-Stokes equations in cylindrical coordinates and they are given by

$$
\frac{\partial^{2} \psi}{\partial r^{2}}-\frac{1}{r} \frac{\partial \psi}{\partial r}+\frac{\partial^{2} \psi}{\partial x^{2}}=\phi(x, r)
$$

with the boundary conditions

$$
\psi(0, r)=f(x), \quad \psi^{\prime}(0, r)=g(x)
$$

Here $\psi$ is stream function and $\phi$ is given function of $x$ and $r$. The Navier Stokes problem requires finding a function $\psi$ which satisfied (15) and the boundary conditions (16). To apply the homotopy perturbation method (HPM), we write (15) in an operator form

$$
L(\psi)+N(\psi)-\phi(x, r)=0
$$

where $L$ and $N$ symbolize $\frac{\partial^{2}}{\partial x^{2}}$ and $\frac{\partial^{2}}{\partial r^{2}}-\frac{1}{r} \frac{\partial}{\partial r}$, respectively.

By the homotopy technique, we construct a homotopy, $\nu(r, p): \Omega \times[0,1] \rightarrow I R$, which satisfies:

$$
\begin{aligned}
H(\nu, p) & =L(\nu)-L\left(\psi_{0}\right)+p L\left(\psi_{0}\right)+p[N(\nu)-\phi(x, r)] \\
& =0, \quad p \in[0,1]
\end{aligned}
$$

where $p \in[0,1]$ is an embedding parameter, while $\psi_{0}$ is an initial approximation of (15), which satisfies the boundary conditions(16). Obviously, from (18) we will have:

$$
\begin{gathered}
H(\nu, 0)=L(\nu)-L\left(\psi_{0}\right)=0 \\
H(\nu, 1)=L(\nu)+N(\nu)-\phi(x, r)=0
\end{gathered}
$$

The changing process of $p$ from zero to unity is just that of $\nu(r, p)$ from $\psi_{0}(x, r)$ to $\psi(x, r)$. In topology, this is called deformation, while $L(v)-L\left(\psi_{0}\right)$ and $L(\nu)+$ $N(\nu)-\phi(x ; r)$ are called homotop.

According to the HPM, we can first use the embedding parameter $p$ as a "small parameter", and assume that the solution of (18) can be written as a power series in $p$ :

$$
\nu=\nu_{0}+p \nu_{1}+p^{2} \nu_{2}+\cdots
$$

Setting $p=1$ yields in the approximate solution of (15) to:

$$
\psi=\lim _{p \rightarrow 1} \nu=\nu_{0}+\nu_{1}+\nu_{2}+\ldots
$$

The combination of the perturbation method and the homotopy method is called the HPM, which eliminates the drawbacks of the traditional perturbation methods while keeping all its advantage.

The series (22) is convergent for most cases. However, the convergent rate depends on the nonlinear operator $L(v)+N(v)$. Moreover, He made the following suggestions [13]: 
(1) The second derivative of $N(v)$ with respect to $\mathrm{v}$ must be small because the parameter may be relatively large, i.e.

(2) The norm of $L^{-1} \partial N / \partial v$ must be smaller than one so that the series converges.

\section{Application}

In this section, we apply the above described methods homotopy perturbation method (HPM), on one examples so that the comparison are made numerically. We wish to emphasize that the purpose of the comparison is only to give the reader insight into the relative efficiencies of the two methods and not definitive comparisons. Also we re-emphasize that the basic intends of new method is user convenience and easy as opposed to speed of computation. In order to verify numerically whether the proposed methodology lead to higher accuracy choose one example which were selected to show the computational accuracy.

\section{Example}

The method is tested on a simple problem of Poisson's equation

$$
\left\{\begin{array}{l}
\frac{\partial^{2} \psi(x, r)}{\partial r^{2}}-\frac{1}{r} \frac{\partial \psi(x, r)}{\partial r}+\frac{\partial^{2} \psi(x, r)}{\partial x^{2}}=4\left(1-r^{2}\right) e^{-2 x} \\
\psi(0, r)=1-r^{2} \\
\frac{\partial \psi}{\partial x}(0, r)=-2+2 r^{2}
\end{array}\right.
$$

According to the HPM, the following homotopy can be constructed:

$$
\begin{gathered}
H(v, p)=\left(\frac{\partial^{2} v(x, r)}{\partial x^{2}}-\frac{\partial^{2} \psi_{0}(x, r)}{\partial x^{2}}\right)+p\left(\frac{\partial^{2} \psi_{0}(x, r)}{\partial x^{2}}-\frac{1}{r} \frac{\partial v(x, r)}{\partial r}\right. \\
\left.+\frac{\partial^{2} v(x, r)}{\partial r^{2}}-4\left(1-r^{2}\right) e^{-2 x}\right)=0
\end{gathered}
$$

Substituting $v$ from equation (21) into equation (24) and some simplification and rearranging based on powers of $p$-terms, we have:

$$
\begin{gathered}
P^{0}: \frac{\partial^{2} v_{0}(x, r)}{\partial x^{2}}-\frac{\partial^{2} \psi_{0}(x, r)}{\partial x^{2}}=0 \\
P^{1}: \frac{\partial^{2} v_{1}(x, r)}{\partial x^{2}}+\frac{\partial^{2} \psi_{0}(x, r)}{\partial x^{2}}-\frac{1}{r} \frac{\partial v_{0}(x, r)}{\partial r}+\frac{\partial^{2} v_{0}(x, r)}{\partial r^{2}} \\
-4\left(1-r^{2}\right) e^{-2 x}=0 \\
P^{2}: \frac{\partial^{2} v_{2}(x, r)}{\partial x^{2}}-\frac{1}{r} \frac{\partial v_{1}(x, r)}{\partial r}+\frac{\partial^{2} v_{1}(x, r)}{\partial r^{2}}=0 \\
P^{3}: \frac{\partial^{2} v_{3}(x, r)}{\partial x^{2}}-\frac{1}{r} \frac{\partial v_{2}(x, r)}{\partial r}+\frac{\partial^{2} v_{2}(x, r)}{\partial r^{2}}=0
\end{gathered}
$$

$$
P^{i}: \frac{\partial^{2} v_{i}(x, r)}{\partial x^{2}}-\frac{1}{r} \frac{\partial v_{i-1}(x, r)}{\partial r}+\frac{\partial^{2} v_{i-1}(x, r)}{\partial r^{2}}=0
$$

To determine $v$, the above equations should be solved. Considering the appropriate initial conditions we have:

$$
\begin{gathered}
v_{0}(0, r)=1-r^{2}, \frac{\partial v_{0}(0, r)}{\partial x}=-2+2 r^{2} \\
v_{i}(0, r)=0, \frac{\partial v_{i}(0, r)}{\partial x}=0, i=1,2, \ldots \\
\psi(x, 0)=\psi_{0}(x, r)=1-r^{2}
\end{gathered}
$$

The solution of equations (25)-(32) may be written as follows:

$$
v_{0}(x, r)=\left(1-r^{2}\right)+x\left(-2+2 r^{2}\right)
$$

$$
v_{1}(x, r)=\left(1-r^{2}\right) e^{-2 x}+2 x\left(1-r^{2}\right)-\left(1-r^{2}\right)
$$

In the same manner, the rest of components was obtained using the maple package. According to the HPM, we can conclude:

$$
\begin{gathered}
\psi(x, r)=\lim _{p \rightarrow 1} v(x, r)=v_{0}(x, r)+v_{1}(x, r) \\
+v_{2}(x, r)+v_{3}(x, r)+\ldots
\end{gathered}
$$

Therefore the solution is:

$$
\psi(x, r)=\left(1-r^{2}\right) e^{-2 x}
$$

Table 1 shows the results obtained by He's HPM method give a good agreement with the Previous numerical results of Greenspan[6], Mittal and Sharma[7] and give the same analytical result found by Bulut and al[8] using Adomian decomposition method.

Finally, it is clear that the application of the He's HPM provide a rapid convergent solution. This scheme has been implemented for wide range of examples and it showed superiority over existing techniques, in particular by determining the exact solution and minimizing the size of the calculations and work.

\section{Conclusion}

In this work, homotopy perturbation method (HPM) has been successfully applied to solve two-dimensional steady flow problem through an orifice, this method was used for nonhomogeneous case of a simple problem of Poisson's equations with initial conditions. The obtained results reveal that the proposed method (HPM) is very simple and straightforward, which avoids the complex procedures of other methods. Furthermore this approach doesn't require any discretization, linearization or small perturbations and therefore is capable of greatly reducing the size of calculations while still maintaining high accuracy of the numerical solution. 
Table 1. The solution of $\psi(x, r)$ for different values of $x$ and $r$

\begin{tabular}{|c|c|c|c|}
\hline$x$ & $r$ & Numerical LAD result & HPM result \\
\hline-2.0 & 0.3 & 0.016679 & 0.016667 \\
0.0 & 0.2 & 0.959986 & 0.960000 \\
0.7 & 0.4 & 0.207159 & 0.207141 \\
-3.0 & 0.8 & $0.145241 \times 10^{3}$ & $0.145234 \times 10^{3}$ \\
-1.0 & 0.7 & $0.376839 \times 10$ & $0.376842 \times 10$ \\
1.5 & 0.7 & 0.025401 & 0.025391 \\
-0.6 & 0.5 & $0.249010 \times 10$ & $0.249009 \times 10$ \\
0.8 & 0.5 & 0.151429 & 0.151422 \\
\hline
\end{tabular}

\section{REFERENCES}

[1] P.J Roache, The LAD, NOS and split NOS methods for the steady state NavierStokes equation, Comput. Fluids, Vol.3, 179-195, 1975.

[2] Parment I.L and Saibel E, Axisymmetric creeping flow from an a orifice in a plane wall, Commun. Pure Appl. Math, Vol.18, 17-23, 1965.

[3] Hunt B.W, Numerical solution of an integral equation for flow froma circular orifice, J. Fluid Mech, Vol. 31, 361-378, 1968.

[4] Gasmi A and Mekias H, The effect of surface tension on the contraction coefficient of a jet, J.Phys. A: Math. Gen Vol. 36, 851-862, 2003.

[5] Gasmi A, Two-dimensional cavitating flow past an oblique plate in a channel, Journal of Computational and Applied Mathematics, http://dx.doi.org/10.1016/j.cam.2013.07.035, 2013.

[6] Greenspan D, Numerical studies of viscous incompressible flow through an orifice for arbitrary Reynolds number, Int. J. Numer. Meth. Eng, Vol.6, 489-496, 1973.

[7] Mittal R.C and Sharma P.K, A one parameter family of LAD methods for the steady state Navier Stokes equations, Comput. Fluids, Vol.13, 507-511, 1985.
[8] Bulut H, Ergüt M, Asil V and Bokor R. H, Numerical solution of a viscous incompressible flow problem through an orifice by Adomian decomposition method, Appl. Math. Comput, Vol.153, 733-741, 2004.

[9] He J H, Comparison of homotopy perturbation method and homotopy analysis method, Applied Mathematics and Computation, Vol.156, 527-539, 2004.

[10] He J H, The homotopy perturbation method for nonlinear oscillators with discontinuities, Applied Mathematics and Computation, Vol.151, 287-292, 2004.

[11] He J H, Application of homotopy perturbation method to nonlinear wave equations, Chaos, Solitons and Fractals, Vol.26, 695-700, 2005.

[12] He J H, Homotopy perturbation method for solving boundary value problems, Physics Letters A, Vol.350, 87-88, 2006.

[13] He J H, Homotopy perturbation technique, Comput. Math. Appl. Mech. Eng, Vol.178, 257-262, 1999. 30 28990 